\title{
Determinants of Neonatal and Post-neonatal Mortality in Pakistan
}

\section{ARSHAD MAHMOOD}

Ensuring the survival and well being of children is a concern of families, communities and nations throughout the world. Since the turn of the 20th century infant and child mortality in more developed countries has steadily declined and, currently, has been reduced to almost minimal levels. In contrast, although infant and child mortality has declined in the past three decades in most less developed countries, the pace of change and the magnitude of improvement vary considerably from one country to another.

The inverse relationship between socio-economic variables of the parents and infant and child mortality is well established by several studies [Muhuri (1995); Forste (1994); Hobcraft, et al. (1984); Caldwell (1979); Sathar (1985, 1987)] and it holds true irrespective of the overall level of mortality in the national populations [Ruzicka (1989)]. The influence of parental education on infant and child health and mortality has proved to be universally significant [Bicego and Boerma (1993); Caldwell, et al. (1990)]. The father's education, mother's education and their work status each have independent effects upon child survival in developing countries [Sandiford, et al. (1995); Forste (1994); Caldwell, et al. (1983)]. Economic conditions of the household also help in explaining the variation in infant and child mortality. The nature of housing, diet, access to and availability of water and sanitary conditions as well as medical attention all depend on the economic conditions of the household. For example, poor families may reside in crowded, unhygienic housing and, thus, suffer from infectious disease associated with inadequate and contaminated water supplies and with poor sanitation [Esrey and Habicht (1986)].

Maternal factors, which are biological attributes of birth, such as the age of mother at the time of childbirth, birth order and birth interval [Forste (1994); Rutstein (1984)], have significant effects on child survival. Infant and child mortality are also affected by the sex of the child, and infants born to mothers who have lost a

M. Arshad Mahmood is currently working as Director in the Ministry of Population Welfare, Government of Pakistan, Islamabad.

Auther's Note: The work presented in this paper was a part of his $\mathrm{PhD}$ dissertation in Sociology, completed at the University of Southern California, Los Angeles. 
child are at greater risk of dying during infancy [Cleland and van Ginneken (1988)]. Moreover, some of the studies found that, within a family, deaths of infants are correlated [Curtis, et al. (1993); Das Gupta (1990); Gubhaju (1985); Majumder (1989)].

Breastfeeding has numerous bio-demographic, social, and economic effects. It affects the health and nutritional status of, both, the mother and child. The role of breastfeeding is very important in the post-neonatal period.

Health seeking behaviour includes both, preventive and curative measures. Preventive measures include immunisation against preventive diseases such as tuberculosis, polio, measles, neonatal tetanus and smallpox, whereas curative measures include the care and types of treatment undertaken for specific conditions, both modern and traditional [Mosley and Chen (1984), p. 28].

In Pakistan infant mortality is still very high and the neonatal mortality contributes more than half of the Infant Deaths. In 1950s it was around 50 percent of the Infant mortality, in 1990s it was about 60 percent of the infant mortality. The Objective of this paper is to identify the factors (proximate determinants such as, demographic factors, environmental factors, nutritional factors and health seeking behaviour) associated with neonatal and post-neonatal mortality by employing Mosley and Chen Framework (1984). This framework represents a complex theoretical paradigm expressed in a simple way, which helps to identify particular determinants of child health and survival. This framework is based on the proposition that socio-economic factors do not directly affect the outcome variable but rather must operate through proximate variables to affect child survival [Mosley and Chen (1984)].

\section{DATA AND METHODOLOGY}

The Pakistan Demographic and Health Survey (PDHS), conducted in 1991, is used to address the objectives of the paper. The primary objective of the PDHS was to provide national as well as provincial-level data on population and health in Pakistan. The PDHS data include a total of 6492 births that occurred 5 years preceding the survey to 4061 women. To avoid the violation of the independence assumption, only the last births are included in the analysis.

Thus, this analysis is restricted to 2132 singleton births, born 1-23 months before the survey. To include the survival status of the older siblings in the analysis, only women are included who have at least two births. Those children share risks associated with family behaviour and childcare practice, such as infant feeding, use of health services and general standards of domestic environment and hygiene. Das Gupta (1990) called them parental competence. Following table depicts the number and percentage of births included in this analysis by each category of reaching at that number. 


\subsection{Conceptual Framework}

Boerma and Bicego (1993) used the conceptual framework proposed by Mosley and Chen (1984) with modifications based on the limitations and structure of the DHS data. Keeping in view the aforementioned frameworks, the model proposed here uses the survival status as the outcome variable. Like the Mosley and Chen (1984) framework, the socioeconomic variables affect the outcome through the four proximate determinants (data on Injury is not available in the DHS) namely, demographic factors, environmental factors, nutritional factors and health seeking behaviour factors. The proposed model to estimate the neonatal and post-neonatal mortality is presented in Figure 1.

The following variables are included in the analysis:

- Socioeconomic Variables: Parental education; household possessions; place of residence; region of residence.

- Demographic Factors: Age of the mother at the time of birth; birth order; birth interval; sex of the child; previous sibling death.

- Environmental Contamination: Source of drinking water; toilet facilities; housing construction material.

- Nutritional Factors: Birth weight; premature birth; nipple feeding;

- Health-seeking Behaviour: Prenatal care; Delivery attendant; place of birth; BCG vaccination and contraceptive use.

In this analysis, Cox-regression is used to estimate the effects of covariates on the neonatal and postnatal mortality. This regression is also called a proportional hazards model. Proportion hazard models have been widely used in demographic research when the time of occurrence of an event is observed.

\subsection{Dependent Variables: Neonatal and Post-neonatal Mortality}

The dependent variable used in the hazard model analysis is neonatal and post-neonatal survival time. It is measured as the duration starting from the infant birth to death, if the event occurred or from the infant birth to the survey date for censored data.

Keeping in view the heaping of age of death at 12 months, the post-neonatal mortality period is collapsed for 1-131 and 12-23 months to avoid biases introduced by any relationships between age at death misreporting and the explanatory variables. Further, only the inclusion of women who had at least two births (excluding first order births), the small number of deaths at older ages and the heavy right-censoring precludes statistically relevant results for a separate child model. Therefore, two observational periods are identified and modeled separately in this analysis: zero completed months (neonatal period); and the period between 1 and 23 months of child age (Post-neonatal mortality). 


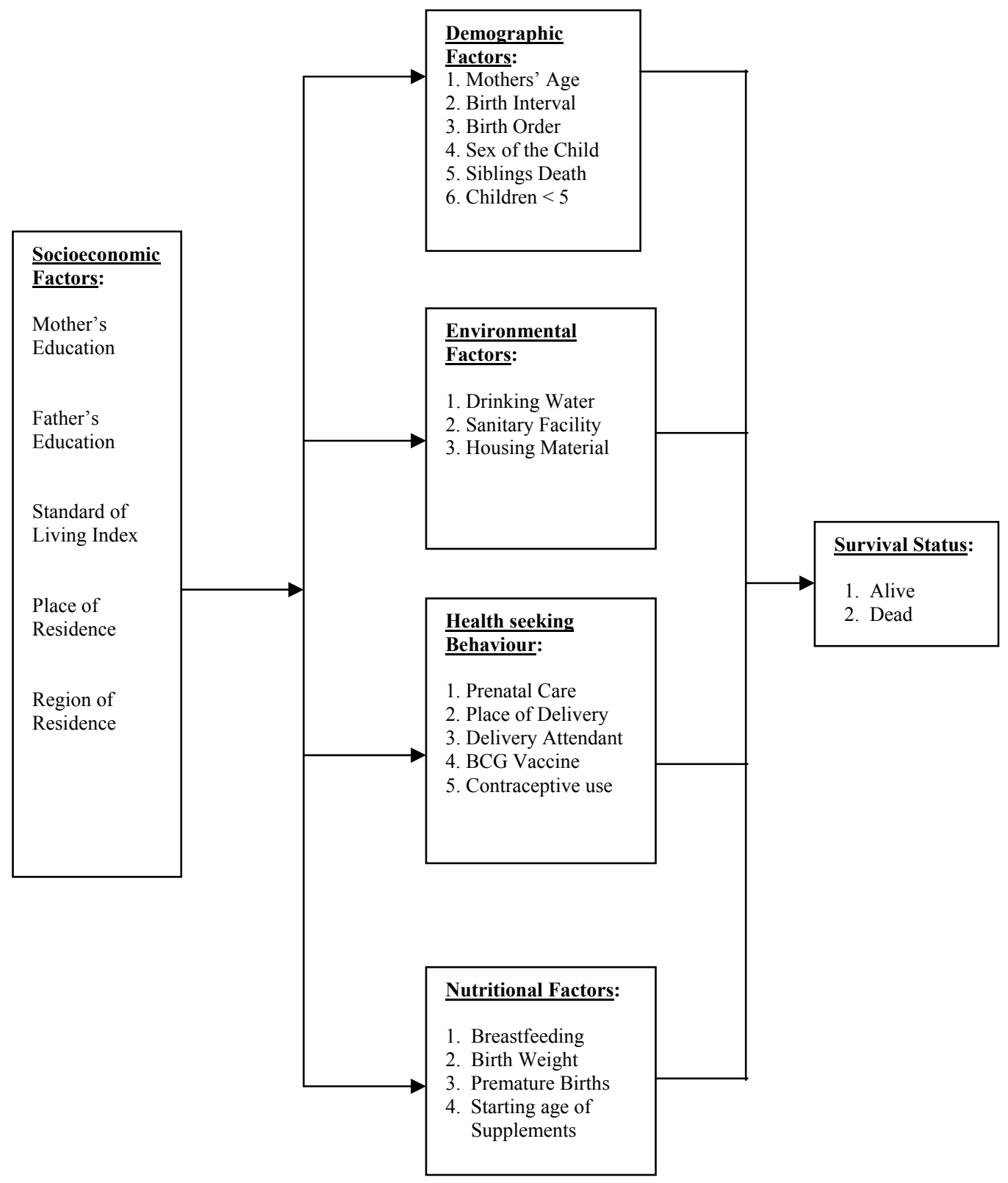

Fig. 1. Proposed Model for Child Survival in Pakistan. 


\subsection{Independent Variables}

\subsubsection{Socioeconomic Variables}

It is assumed that the socioeconomic conditions of the families remained the same during the five years period before the survey. Therefore, the household conditions when the children were born assumed to be the same as when the data were collected.

\section{Index of Household Possessions}

In the absence of income data, the index of household possessions was used as a proxy for family wealth. The household resources are indicated by possession of household goods such as, refrigerator, television, radio, bicycle and motorcycle. From these indicators, an index was created by summing-up these scores to represent the economic position of the household by assigning an equal score to each of these items. A maximum score of 5 indicates a high socioeconomic status and a very low socioeconomic status by having a 0 score. Then it was categorised into three: low $(0$ score), medium (1-2 score) and high (3-5 score) socioeconomic status.

\section{Parental Education}

The mothers' education is important in studying the survival status of children. The mothers' education affects the outcome through different ways as studied by many researchers. It is commonly assumed that greater empowerment and autonomy are inevitable consequences of schooling and that these are the main pathways that link education to better child survival.

In Pakistan, female literacy is very low. Female education has generally lagged behind male education due to a comparatively unfavourable societal attitude. The major bottleneck to formal education is lack of schools for females, especially in rural areas. Since education is sex-segregated in the country, female teachers are reluctant to work in isolated rural areas or even in urban slum areas [Pakistan (2000)].

\section{Place of Residence}

The place of residence of the parents affects, both, the survival status and nutritional status of the living children in developing countries. The urban areas are mostly equipped with a better infrastructure for health services than rural areas. Availability and accessibility to public health services at the community level play a crucial role in child survival prospects. In most developing countries, availability of primary health services is mostly concentrated in urban cities where people with higher socioeconomic status tend to reside. In rural areas, health centres are generally characterised by poor services and staffing. In Pakistan, both the public and private sector are providing medical facilities in the country but the private sector has concentrated in the urban areas. 


\subsubsection{Household Environment and Hygiene}

Source of Drinking Water

Safe and clean drinking water is important for human health, especially for children whose immune systems are still maturing. Even if contaminated water is used for washing or bathing, it increases the chances of catching infection. When supplementary foods are prepared for the infants and children, use of clean water is very important. If the water is contaminated, it is more likely that the child will get infections and diarrhea. It has been reported that poor or contaminated sources of drinking water are associated with an increased number of deaths due to diarrhea. The access to piped water in the household is likely to be of most direct benefit in lowering early childhood mortality by reducing exposed to water-borne diseases, particularly diarrhea and dysentery.

In this analysis, five sources of water supply are included: piped water into the residence; piped water on to the property; public tap; well and surface water (such as, river or rain).

\section{Toilet Facilities}

Toilet facilities are very important for the health and hygiene for, both, children and adults. Proper use of flush toilets can reduce risk of parasite infection, morbidity and poor nutrition status of children.

In Pakistan, the majority of children are not diapered, but are allowed to urinate and defecate freely on the dirt floor (most of the houses do not have cemented floor), after which the mother or the older sister sweeps the excrement out into the yard. It is assumed that households with flush toilets are hygienically better than those which have other source of toilet facilities.

Three different sources of toilet facilities are included in this analysis. Flush system; latrine (pit and bucket); no facilities (they do not have any facility at home, use nearby crops or bushes).

\section{Housing Material}

Material used in building the house was also included in the survey. The construction shows the hygienic conditions of the building. Houses built of baked bricks (cemented) are supposed to be hygienically better than the houses constructed from mud or unbaked bricks.

\subsubsection{Demographic and Maternal Factors}

\section{Preceding Birth Interval}

It is a well established fact that infant and child mortality varies by the length of the preceding birth interval. Therefore, three different dummies of preceding birth 
intervals are included in this analysis: 18 months and less; 19-35 months; 36-47 months; and over 48 months.

\section{Birth Order}

Birth order is included in this analysis because birth order not only tells us the rank of the child in the family but also tells us something about the number of children in the family. In households with limited resources, its distribution depends on the number of children in the household. The larger number of children indicates a smaller share of scarce resources. Because the children have to compete for their share, the nutritional requirements for growing children may result in malnutrition.

In most of the developing countries, higher mortality risks were found for the first-born children compared to birth order two through six [Sullivan, et al. (1994); Rutstein (1984); Hobcraft, et al. (1985)].

\section{Previous Death of Siblings}

The idea that children of certain families have biological traits which predispose them to high mortality is supposed by evidence that the effects of sibling deaths on the health of the subsequent child are considerably significant even after controlling for socioeconomic, biological and behavioural factors [Curtis, et al. (1991); Das Gupta (1990); Hobcraft, et al. (1985)]. The survival status of the previous child is observed to be associated with the length of the birth interval as well as with breastfeeding status.

In this analysis, the survival status of the previous child is included to see if that has any effect on the survival status of the index child in Pakistan. To include this variable in the analysis, we need at least two births. Therefore, this variable is obviously not applicable the first order born children in the analysis.

A previous death is defined as a dichotomous variable indicating if death occurred coded as 1 , elsewhere coded as 0 .

\subsubsection{Nutritional Status /Dietary Intake}

\section{Birth Weight/ Birth Size}

In Pakistan, a large proportion of births occur at home and it was difficult to obtain the exact birth weights of these babies. In the PDHS, a question asked was: was the baby very large, large, medium, small or very small at the time of the birth. This is clearly far from a birth weight, but it would be meaningful if the mother herself evaluates her baby as being very large or very small than average. Low birth weight poses a greater threat to a child born in conditions prevailing in slums or villages of developing countries than to a child born in a society with adequate medical services and a satisfactory physical and cultural environment [Kimm (1979)]. Babies born very big are abnormal babies and their risk of mortality during 
neonatal period is also higher than the normal size born babies [Rees, et al. (1999)]. Two dummy variables are created, one for very small and the second for very large keeping small, medium and large as reference.

Premature birth with its consequent low birth weight is one of the outcomes of that has been described as the "maternal depletion syndrome". It is known to be associated with maternal age $(<20$ and $>35)$, short birth interval [Carlaw, et al. (1983)], high birth order [Hobcraft, et al. (1985)] and a higher risk of death.

\section{Breastfeeding}

Breastfeeding is used as a time-variant covariate for the analysis of postneonatal mortality. However, for the small number of children who died in the first month of life and who did not have the chance to breastfeed, death is not associated with lack of breastfeeding. The dummy is coded as 1 for all infants who died in the first month along with all other children who breastfed during the first year of their life. This procedure will produce a small conservative bias in estimating the effect of breastfeeding on child survival.

\section{Supplementary Food}

The period when the children's health is most in danger starts at three months and lasts until they can feed themselves, at about three years old. During this period several feeding practices can have an influence on the child's nutrition. First, is the age at which food supplements are introduced into the child's diet and second, is the method of preparation of the supplementary food.

The prevalence of bottle-feeding with nipples is another possible source of contamination of milk and provides an indication of inappropriate feeding practice [Phillips, et al. (1969)]. Studies in developing countries found bottles and nipples were contaminated by different pathogens [Elegbe, et al. (1982); Black, et al. (1989)]. Improved cleaning of bottles and nipples may reduce the risk of contamination of milk or formula by boiling of bottles and nipples. However, proper use of these procedures is often difficult because poor households may have only one feeding bottle which is constantly in use. In this analysis, supplementary food is also used as a time-varying variable.

\subsubsection{Health Seeking Behaviour}

\section{Prenatal Care}

Regular prenatal care is needed to help detect and manage some pregnancyrelated complications and to educate women about danger signs, potential complications, and where to seek help. Prenatal care beginning early in the first trimester of pregnancy and continuing on a regular basis is important to the health of both mother and infant. Early prenatal care provides an opportunity to offer 
preventive care that will benefit the infant as well as the mother such as, counseling on hygiene, breastfeeding, nutrition, family planning, tetanus toxoid immunisation and iron and folate supplementation. Prenatal care also benefits treatment of existing diseases that may be aggravated by pregnancy. Prenatal care helps to prevent complications during pregnancy and labour. Also, there is a negative association between timing of prenatal care and low birth-weight. If a woman's pregnancy goes to term, she may typically have anywhere from 10 to 14 prenatal visits. In PDHS, if the respondent reported prenatal care was taken, then the question regarding the number of visits were also asked.

\section{Place of Delivery}

It is a fact that deliveries at medical institutions are safer, both, for mother and the child. Hence, a higher child survival rate is expected for these deliveries. The assumption is that mothers who have delivered in hospitals will also be aware of better child health care practices. These include the importance of breastfeeding, hygiene, nutrition, immunisation etc. Moreover, hospitals are assumed to maintain safe delivery environment, and newborns may have fewer chances of contamination. Hence, these children should be better off than children born at home.

Although delivery services are almost free in government hospitals/clinics, some people prefer private clinics/doctors for several reasons. First, it is generally believed that anything worthwhile or valuable will cost money. The medical services that must be paid for are seen as better than government services. In addition, practitioners who charge for these services are expected to be more polite and attentive and to devote more care and concern to patients.

Three dummies are included to represent this variable: one for delivery at a governmental hospital; second a dummy for delivery at a private hospital; and the third dummy for if delivery occurred at other than respondents' home keeping at respondents home as reference.

\section{Birth Attendant}

A birth attended by trained medical staff such as physicians, nurses, or family planning workers would be safer for both mother and the newborn infant. These medically trained personnel are more likely to use sterilised equipment for the delivery compared to the non-trained personnel. Hence, this variable is categorised 1 if the birth was attended by the trained medical staff and 0 elsewhere.

\section{BCG Vaccination}

Vaccination against tuberculosis prevents a disease that carries an increased risk of dying for children in developing countries. According to the Global Tuberculosis Control WHO Report 2000, in Pakistan about 1.5 million people suffer 
from TB, and more than 210,000 new cases occur each year. BCG is included in the analysis as a proxy of immunisation on the assumption that if a baby get the BCG it is more likely that the child will get the complete immunisation course.

\section{RESULTS}

In this section, results of proportional hazard analyses of neonatal and postneonatal mortality are presented. The exponential function of each regression coefficient shown in the tables represents the relative risk rates.

\subsection{Neonatal Mortality}

Table 1 depicts the multivariate results of the proportional hazard models in relation to neonatal mortality. The results are shown in the form of hazard risk ratios and the significance level. Among the socioeconomic factors in model-1, children living in the provinces of Punjab $(R R=1.76, p<0.10)$ and $\operatorname{Sindh}(R R=2.08, p<0.05)$ have significantly higher neonatal mortality compared to the children living in NWFP and Balochistan provinces (Reference). When the environmental factors are added in model-2, it is observed that the neonatal mortality difference in living in Punjab disappeared. However, residence in the Sindh province still has a significantly higher neonatal mortality. When demographic variables are included in the model, it is seen that the maternal age over 30 years, previous birth intervals less than 18 months, number of children under age 5 years, and previous sibling death have positive significant effects in increasing the risk of neonatal mortality.

In model-4, when the baby-size and premature births are included, both premature births and baby-size at birth have significant effects on neonatal mortality. Premature born babies have 13.6 times higher risk of neonatal mortality than fullterm born babies. Babies of very-large size also have more than 8.5 times higher risk of neonatal mortality than normal-sized born babies. Another interesting change to note is that the significance of higher neonatal mortality in the province of Sindh was spurious. The higher neonatal mortality in Sindh was due to higher proportion of premature births and babies of very large-sized at birth. The neonatal mortality in the province of Punjab is slightly increased, however, it is not significant.

Including health seeking behaviour variables in model-5, it is seen that babies who had BCG vaccine at birth are at a significantly lower risk of neonatal mortality than those who did not receive BCG immunisation at birth. The utilisation of other preventive health services, such as prenatal care and delivery attended by a medical personnel, did not show any significant effect on neonatal mortality. However, babies delivered at government hospitals maintained a higher risk of neonatal mortality than babies delivered at home $(\mathrm{p}<0.10)$. Again, including the health seeking variables in the model, it is observed that the effect of Sindh reduced and the Punjab increased. 
Table 1

Hazard Rate Ratios Obtained from Proportional Hazard Model for Predicting Neonatal Mortality, PDHS, 1990-91

\begin{tabular}{|c|c|c|c|c|c|c|}
\hline Independent Variables & Model-1 & Model-2 & Model-3 & Model-4 & Model-5 & Model-6 \\
\hline Years of Maternal Education & 0.920 & 0.927 & 0.945 & 0.897 & 0.941 & 0.933 \\
\hline Years of Paternal Education & 1.005 & 1.008 & 1.008 & 1.009 & 1.010 & 1.012 \\
\hline \multicolumn{7}{|c|}{ Household Possessions Index: Lower (Ref) } \\
\hline Higher & 0.565 & 0.612 & 0.508 & 0.478 & 0.557 & 0.623 \\
\hline Medium & 0.811 & 0.813 & 0.851 & 0.807 & 0.882 & 0.894 \\
\hline Place of Residence: Urban & 0.793 & 1.009 & 1.192 & 1.243 & 1.388 & 1.050 \\
\hline \multicolumn{7}{|c|}{ Province of Residence: NWFP and Balochistan (Ref) } \\
\hline Punjab & $1.760+$ & 1.655 & 1.304 & 1.455 & 1.830 & $2.101+$ \\
\hline Sindh & $2.080^{*}$ & $2.059 *$ & 2.090 & +1.347 & 1.294 & 1.448 \\
\hline \multicolumn{7}{|c|}{ Source of Drinking Water: Well (Ref) } \\
\hline Pipe in to the House & & 0.707 & 0.648 & 0.743 & 0.666 & 0.736 \\
\hline Pipe on to the Property & & 0.534 & 0.554 & 0.526 & 0.646 & 0.695 \\
\hline Public Tap & & 0.670 & 0.562 & 0.649 & 0.715 & 0.782 \\
\hline Surface & & 0.605 & 0.557 & 0.577 & 0.488 & 0.721 \\
\hline \multicolumn{7}{|c|}{ Latrine Facility: No Facility (Ref) } \\
\hline Bucket or Pit & & 1.827 & 1.791 & 1.596 & 1.300 & 1.896 \\
\hline Flush & & 1.585 & 1.516 & 1.584 & 1.038 & 1.205 \\
\hline Construction Baked Bricks & & 1.335 & 1.048 & 1.181 & 0.936 & 0.928 \\
\hline \multicolumn{7}{|l|}{ Mother Age: 20-29 (Ref) } \\
\hline $15-19$ & & & 0.677 & 0.746 & 0.822 & 0.710 \\
\hline $30-49$ & & & $2.771 * * *$ & $2.419 * * *$ & $2.354 * * *$ & $2.514 * * *$ \\
\hline \multicolumn{7}{|c|}{ Previous Birth Interval: 36 - 47 (Ref) } \\
\hline Less than 18 Months & & & $3.147 * * *$ & $2.992 * * *$ & $3.698 * * *$ & $10.114 * * *$ \\
\hline $18-35$ & & & 1.445 & 1.187 & 1.513 & $3.246^{* *}$ \\
\hline 48 and More & & & 0.607 & 0.689 & 0.783 & 0.741 \\
\hline
\end{tabular}


Table 1-(Continued $)$

Birth Order: 2nd (Ref)

3 rd -5 th

6th and above

Sex of the Child:Boy

Number of Children under 5

Sibling Death

Premature Birth

Baby-size at Birth:Average (Ref)

Small-size

Big-size

Prenatal Care Visits

Place of Delivery: At Home (Ref)

At Government Hospital

At Private Hospital

At Home other than Mother's

Delivery Attended by Medical Personal

BCG Vaccine received

Contraceptive Use

Interactions:

Urban and Prenatal Care

Space $<18$ and Prenatal Care

Space 18-35 and Prenatal Care

Log Likelihood

Degrees of Freedom

$\begin{array}{llll}1.557 & 1.454 & 1.213 & 1.356 \\ 0.442^{* * *} & 0.523^{* *} & 0.555^{*} & 0.462^{* * *} \\ 0.979 & 0.937 & 1.054 & 1.102 \\ 1.561^{* *} & 1.598^{* *} & 1.468^{*} & 1.496^{*} \\ 3.400^{* * *} & 2.783^{* * *} & 2.484^{* * *} & 7.901^{* * *} \\ & 13.648^{* * *} & 6.442^{* * *} & 6.566^{* * *} \\ & & & \\ & 0.974 & 0.889 & 0.981 \\ & 8.557^{* * *} & 4.601^{* * *} & 4.714^{* * *} \\ & & 0.982 & 0.699^{*} \\ & & & \\ & & 2.546+ & 2.843^{*} \\ & & 1.921 & 2.154 \\ & & 1.812 & 2.146 \\ & & 0.692 & 0.739 \\ & & 0.017^{* * *} & 0.016^{* * *} \\ & & 1.057 & 0.867\end{array}$

*** $\mathrm{p}<0.001, * * \mathrm{p}<0.01, * \mathrm{p}<0.05,+\mathrm{p}<0.10$. 
In this analysis, it is observed that demographic, nutritional and health seeking behaviour factors are responsible for higher neonatal mortality in Pakistan. The higher neonatal mortality observed in Sindh was due to the higher proportion of premature births and the lower utilisation of health services.

To test if any behavioural factors may also affect the outcome of pregnancy other than the biological mechanisms operating in the prenatal period, the interactions between demographic and health care variables are included in model-6.

It is hypothesised that pregnant mothers with short spacing of births still have very young children and may not take prenatal care services that would otherwise be the case. On the other hand, pregnant women with longer birth intervals are more likely to attend prenatal care services which ultimately results in a healthy child birth [Boerma and Bicego (1992)]. When the interactions between prenatal care and previous birth intervals are included in the model, several changes in the significance of other variables are also noted.

First of all, this analysis supports the argument that, if mothers with shorter previous birth intervals have used prenatal care, their babies are significantly more likely to have better survival chances during the neonatal period than those mothers with the same short birth interval who did not receive prenatal care for the index child. The hazard analysis shows that the babies of short previous birth intervals have statistically significantly improved chances of survival during the neonatal period if their mothers had attended the prenatal care. It is also observed that babies delivered at government hospitals have significant higher neonatal mortality than babies delivered at respondents own home even after controlling for all other variables.

The interaction model also shows that babies of mothers living in rural areas, and who received prenatal care, have significantly higher chances of survival during neonatal period than their counterparts living in urban areas. The results clearly demonstrate the importance of prenatal care in rural areas. If mothers go for prenatal care to check-up and to help detect and manage some pregnancy-related complications and to educate themselves about danger signs, potential complications, and where to seek help. Early prenatal care provides an opportunity to offer preventive care that will benefit the infant as well as the mother such as, counseling on hygiene, breastfeeding and nutrition.

\subsection{Post-neonatal Mortality}

Table 2 shows the results of proportional hazard analysis in model-1 through model-5. Model-1, which includes all the socioeconomic variables shows that fathers' education has a negative significant effect on post-neonatal mortality $(\mathrm{RR}=0.91, \mathrm{p}<0.01)$. The children of parents living in the province of Sindh $(\mathrm{RR}=2.41, \mathrm{p}<05)$ also shows a significant higher post-neonatal mortality compared to the children living in the provinces of NWFP and Balochistan. 
Table 2

Hazard Rate Ratios Obtained from Proportional Hazard Model Analysis for Predicting Post-neonatal Mortality, PDHS, 1990-91

\begin{tabular}{|c|c|c|c|c|c|}
\hline Independent Variables & Model-1 & Model-2 & Model-3 & Model-4 & Model-5 \\
\hline Years of Maternal Education & 1.012 & 1.017 & 1.033 & 1.029 & 1.069 \\
\hline Years of Paternal Education & $0.912 * *$ & $0.909^{* *}$ & $0.917^{* *}$ & $0.923 *$ & $0.912 * *$ \\
\hline \multicolumn{6}{|c|}{ Household Possessions Index: Lower (Ref) } \\
\hline Higher & 0.931 & 0.905 & 0.731 & 0.691 & 0.848 \\
\hline Medium & 1.031 & 0.990 & 0.870 & 0.841 & 1.026 \\
\hline Place of Residence: Urban & 0.816 & 0.812 & 0.825 & 0.813 & 0.895 \\
\hline \multicolumn{6}{|c|}{ Province of Residence: NWFP and Balochistan (Ref) } \\
\hline Punjab & 1.702 & 1.684 & 1.354 & 1.247 & 1.361 \\
\hline Sindh & $2.414^{*}$ & $2.706^{* *}$ & $2.850 * *$ & $2.729 *$ & $2.770^{*}$ \\
\hline \multicolumn{6}{|c|}{ Source of Drinking Water: Well (Ref) } \\
\hline Pipe in to the House & & $0.339 *$ & $0.301 * *$ & $0.297^{* *}$ & $0.296^{*}$ \\
\hline Pipe on to the Property & & 0.737 & 0.667 & 0.660 & 0.605 \\
\hline Public Tap & & 0.770 & 0.685 & $0.665+$ & 0.666 \\
\hline Surface & & $0.369+$ & $0.324 *$ & 0.359 & $0.310+$ \\
\hline \multicolumn{6}{|c|}{ Latrine Facility: No Facility (Ref) } \\
\hline Bucket or Pit & & 1.371 & 1.409 & 1.502 & 1.561 \\
\hline Flush & & 1.436 & 1.609 & 1.819 & 1.869 \\
\hline Construction Baked Bricks & & 1.148 & 1.120 & 1.141 & 1.063 \\
\hline \multicolumn{6}{|l|}{ Mother Age: 20-29 (Ref) } \\
\hline $15-19$ & & & 0.170 & 0.173 & $0.128+$ \\
\hline $30-49$ & & & $2.575^{* * *}$ & $2.721 * * *$ & $2.545^{* * *}$ \\
\hline \multicolumn{6}{|c|}{ Previous Birth Interval: 36 - 47 (Ref) } \\
\hline Less than 18 Months & & & $2.062+$ & $2.082+$ & $2.378^{*}$ \\
\hline $18-35$ & & & $2.358 * *$ & $2.260 * *$ & $2.386 * *$ \\
\hline 48 and More & & & 0.920 & 0.937 & 0.997 \\
\hline
\end{tabular}


Table 2-(Continued)

Birth Order:

2nd

6th and above

Sex of the Child: Boy

Number of Children under 5

Previous Sibling Death

Premature Birth

Baby-size at Birth: Average (Ref)

Small-size

Big-size

Breastfeeding

Age at Supplementary Food Started

Feeding with Nipple

1.596

$0.519^{*}$

0.868

$1.413+$

$4.448 * * *$

Place of Delivery: At Home (Ref)

At Government Hospital

At Private Hospital

Delivery Attended by Medical Persona

BCG Vaccine received

Contraceptive used

Log likelihood

Degrees of Freedom

p $<0.01, * p<0.05,+p<0.10$.

\subsection{5}

$0.519 *$

1.875
$0.527 *$

0.822

$\begin{array}{ll}1.428+ & 1.462 * \\ 4.284 * * * & 4.492 * * *\end{array}$

$3.667 * \quad 4.940^{* *}$

$0.801 \quad 0.676$

$0.528 \quad 0.406$

$0.349 * * \quad 0.346^{* *}$

$4.066^{* * *} \quad 4.143^{* *}$

0.956

1.140

1.140

0.953

0.805

$0.304 * * *$

1.874

172.09 
When environmental variables are added in model-2, along with the socioeconomic variables, it is observed that children living in households connected with "piped water in to the house" $(\mathrm{RR}=0.34, \mathrm{p}<0.05)$ and households depending on surface water have reduced risks of post-neonatal mortality compared to the children living in households with a well source of drinking water.

In model-3 when the batch of demographic variables are added to socioeconomic and environmental variables, older maternal age, shorter previous birth interval, lower birth order, and the previous sibling death in a family all have a positive significant effect on post-neonatal mortality. However, the fathers' education and piped water inside the households still maintain their significance even after controlling for all other socioeconomic, environmental, and demographic variables in the model.

In the next model when the nutritional factors, including baby-size and premature births are added, premature births have higher risks of post-neonatal mortality even after controlling for other variables. Breastfeeding $(R R=0.35, p<$ $0.01)$ and age of non-supplementary food $(R R=4.07, p<0.001)$ are highly significant in predicting the post-neonatal mortality.

Including health seeking behaviour variables in model-5, it is observed that only babies who had BCG vaccine at birth are at a significantly lower risk of postneonatal mortality than those who did not receive BCG immunisation at birth. The utilisation of other preventive health services, such as prenatal care or delivery attended by medical personnel does not show any significant effect on post-neonatal mortality.

In the analysis where all the variables are included in the multivariate model, it is observed that among the socioeconomic variables, the fathers' education $(\mathrm{RR}=0.91, \mathrm{p}<0.01)$ has significantly reduces post-neonatal mortality. Residence in the province of Sindh is also associated with higher post-neonatal mortality. Among the environmental variables, piped water connected in to the house maintained its importance to significantly improve the survival status of children during the postneonatal period. Maternal age over 30 years $(\mathrm{RR}=2.55, \mathrm{p}<0.001)$, birth intervals of less than 18 months $(\mathrm{RR}=2.38, \mathrm{p}<0.05)$, previous birth interval 18-35 months $(\mathrm{RR}=2.40, \mathrm{p}<0.01)$, and siblings death $(\mathrm{RR}=4.49, \mathrm{p}<0.001)$, are associated with higher post-neonatal mortality.

As mentioned earlier, breastfeeding proved its importance during the first two years of a child's life. Hazard models show the significant effect $(R R=0.346, p<$ 0.01 ) of continued breastfeeding on reducing post-neonatal mortality. Breastfeeding reduces post-neonatal mortality by more than 62 percent, keeping constant the effect of all other variables. The age of start giving supplementary food is also very important in predicting the post-neonatal mortality. The proportional hazard model shows that as the age of non-supplementary food is delayed, the risk of post-neonatal mortality increases by 4 fold if the supplementary food started later in childhood $(\mathrm{RR}=4.14, \mathrm{p}<0.001)$. 
When the interactions between prenatal care and previous birth intervals are included in the next model, several changes in the significance of other variables are also noted. The advantage is that attending prenatal care for mothers with shorter birth intervals still improves the survival status during the post-neonatal period. The interaction model shows that if mothers with shorter previous birth intervals have used prenatal care, their babies are significantly less likely to die during the postneonatal period than those mothers with the same short birth interval who did not receive prenatal care for the index child. The same significant impact of prenatal care for short birth interval babies was also observed for neonatal mortality.

In Table 3 when the interaction between bottle feeding with nipple and maternal education is added to test the hypothesis that educated mothers are more likely to clean the bottle and nipple before giving it to the child than their uneducated counterparts. The result of this interaction provided the evidence that nipple improves the survival status of the children of educated mothers. In this analysis, the nipple feeding shows the non-significant negative effect on post-neonatal mortality in the additive model but in the interaction model it shows a negative significant $(\mathrm{RR}=0.87, \mathrm{p}<0.10)$ effect on post-neonatal mortality for educated mothers.

Earlier research indicates that bottle-feeding with nipples is a possible source of contamination of milk and provides an indication of inappropriate feeding practice [Phillips, et al. (1969)]. Studies in developing countries found bottles and nipples were contaminated by different pathogens [Elegbe, et al. (1982); Black, et al. (1989)].

The interaction model also shows that infants of higher socioeconomic status enjoy higher survival chances during the post-neonatal period. However, if the numbers of children under 5 years are more than 1 the chances of post-neonatal mortality significantly increases for these children of higher index households $(\mathrm{RR}=4.55, \mathrm{p}<0.001)$.

\section{CONCLUSION}

Contrary to the common perception that maternal education is a more important determinant of infant mortality than paternal education, the results of PDHS suggest that father's education is a better predictor of post-neonatal mortality than maternal education. Maternal education is associated with reduced postneonatal mortality in rural areas. Bicego and Boerma (1993) also found urban-rural differentials in child health and argued that advantage of maternal education is more pronounced in rural areas than in urban areas. Maternal education also play significant role in hygienically feeding bottle milk compared to their uneducated counterparts which improve the survival status after neonatal period.

Children of mothers with a higher index of household possessions are also at significantly lower risk of post-neonatal mortality. However, the proportional hazard 
Table 3

Odds Ratios Obtained from Proportional Hazard Model Analysis for Predicting Post-neonatal Mortality, PDHS, 1990-91

\begin{tabular}{|c|c|c|}
\hline Independent Variables & Model-6 & Model-7 \\
\hline Years of Maternal Education & 0.944 & 0.955 \\
\hline Years of Paternal Education & $0.911 * *$ & $0.915^{*}$ \\
\hline \multicolumn{3}{|l|}{ Household Possessions Index: Lower (Ref) } \\
\hline Higher & $0.026 * * *$ & $0.032 * * *$ \\
\hline Medium & 0.974 & 1.040 \\
\hline Place of Residence: Urban & 0.713 & 1.009 \\
\hline \multicolumn{3}{|l|}{ Province of Residence: NWFP and Balochistan (Ref) } \\
\hline Punjab & 1.525 & \\
\hline Sindh & $2.695^{*}$ & $2.074 *$ \\
\hline \multicolumn{3}{|l|}{ Source of Drinking Water: Well (Ref) } \\
\hline Pipe in to the house & $0.314 * *$ & $0.357 *$ \\
\hline Pipe on to the Property & 0.877 & 0.983 \\
\hline Public Tap & 0.746 & 0.709 \\
\hline Surface & $0.336 *$ & $0.338 *$ \\
\hline \multicolumn{3}{|l|}{ Latrine Facility: No Facility (Ref) } \\
\hline Bucket or Pit & 1.723 & \\
\hline Flush & 2.203 & \\
\hline \multicolumn{3}{|l|}{ Mother Age: 20-29 (Ref) } \\
\hline $15-19$ & 0.129 & $0.126+$ \\
\hline $30-49$ & $2.787 * * *$ & $2.811 * * *$ \\
\hline \multicolumn{3}{|l|}{ Previous Birth Interval: 36 - 47 (Ref) } \\
\hline Less than 18 months & $6.413 * * *$ & $6.364 * * *$ \\
\hline $18-35$ & $5.357 * * *$ & $5.393 * * *$ \\
\hline 48 and more & 0.864 & 0.850 \\
\hline \multicolumn{3}{|l|}{ Birth Order: 3-4 (ref) } \\
\hline $2^{\text {nd }}$ & 1.939 & 2.089 \\
\hline 6th and above & $0.486 * *$ & $0.482 * *$ \\
\hline Sex of the Child: Boy & 0.867 & \\
\hline Number of Children under 5 & 1.248 & 1.239 \\
\hline Previous Sibling Death & $10.863^{* * *}$ & $11.047 * * *$ \\
\hline Premature Birth & $5.457 * *$ & $5.262 * *$ \\
\hline Breastfeeding & $0.349 * *$ & $0.367 * *$ \\
\hline Age at Supplementary food started & $4.207 * * *$ & $4.033 * * *$ \\
\hline Feeding with Nipple & 0.957 & 1.033 \\
\hline Prenatal Care Visits & 0.951 & 0.951 \\
\hline At Government Hospital & 1.244 & \\
\hline At Private Hospital & 1.038 & \\
\hline Delivery Attended by Medical Personal & 0.762 & \\
\hline BCG Vaccine received & $0.337 * * *$ & $0.341^{* * *}$ \\
\hline Contraceptive used & 1.203 & \\
\hline \multicolumn{3}{|l|}{ Interactions } \\
\hline Prenatal Care and Spacing Less than 18 Months & $0.208^{*}$ & $0.206^{*}$ \\
\hline Prenatal Care and Spacing 19-35 Months & $0.251^{*}$ & $0.242 * *$ \\
\hline Higher Index and Under 5 Children & $4.728 * * *$ & $4.552 * * *$ \\
\hline Mother Education and Urban Residence & $1.298+$ & $1.289+$ \\
\hline Mother Education and Feeding with Nipple & $0.873+$ & $0.873+$ \\
\hline Log likelihood & 197.047 & 190.832 \\
\hline Degrees of Freedom & 38 & 30 \\
\hline
\end{tabular}

*** $\mathrm{p}<0.001, * * \mathrm{p}<0.01, * \mathrm{p}<0.05,+\mathrm{p}<0.10$ 
analysis shows that among the socioeconomic factors only maternal education at bivariate level and region of residence affect neonatal mortality in Pakistan.

The results of proportional hazard model analysis also show that families living in households connected with piped water in their houses have significantly lower post-neonatal mortality than those families who depend on wells for drinking water. The results are indicative of the importance of safe drinking water for improving post-neonatal mortality in Pakistan. The results do not find evidence of improved child survival in households who have flush toilet facilities than those who do not.

Among the demographic variables, it is observed that children of older women (30-49) are exposed to significantly higher neonatal and post-neonatal mortality. On the other hand, babies of the youngest mothers are at the lower risk of both neonatal and post-neonatal mortality. The explanation for this is that, in this analysis, the index children are not the first order babies of these teenage mothers. In the earlier research it was observed that children of younger mothers are at higher risk of infant and child mortality because in most of the cases they were the first order babies. Children born with shorter previous birth intervals are also at significantly higher risk of neonatal and post-neonatal mortality. A short birth interval does not give the mother sufficient time to recuperate from the birth and to replenish her stores of nutrients used during pregnancy, especially in conditions of malnutrition.

The analysis highlighted the advantage of prenatal care to prevent these neonatal and post-neonatal deaths if these mothers avail the prenatal care services when they got pregnant after shorter intervals. The hazard analysis also shows that risk of both neonatal and post-neonatal mortality for those babies who has already lost own sibling are significantly higher than the babies who did not experience a previous sibling death. This may be that the sibling deaths tend to be correlated due to the same risks associated with the home environment and with their mother's health and reproductive behaviour.

Among the nutritional factors, longer breastfeeding, and early start of supplementary foods are associated with higher survival during the post-neonatal period. Premature born babies are at higher risk of death both during the neonatal and post-neonatal periods. The analysis also shows that large-sized babies are at significantly higher risk of neonatal deaths but if they survived the neonatal period then these big-sized born babies have better chances of survival than average-sized born babies.

Vaccination of BCG at birth is an important predictor of lower neonatal and post-neonatal mortality in Pakistan. It is also observed that children living in the province of Punjab have higher neonatal mortality than children living in the other provinces whereas, children living in the province of Sindh have significantly higher post-neonatal mortality than the children living in any other province. 
Hence, on the basis of these results, it can be suggested that the rise in the parental education, improvements in the quality of water supply and motivation of mothers to utilise the health services for prenatal and post natal care including immunisation are the most important steps to be taken to reduce the differentials in neonatal and post-neonatal mortality in Pakistan. The analysis shows that the causes of mortality after neonatal period are deeply rooted in poverty, unsafe drinking water, non-utilisation of health services and lack of parental education. The analysis also shows that child mortality can significantly be reduced if the interval between births be expanded by 3-4 years. Use of family planning methods for spacing purposes definitely help in reducing both neonatal and post-neonatal mortality in Pakistan.

\section{REFERENCES}

Bicego, George T., and J. Ties Boerma (1993) Maternal Education and Child Survival: A Comparative Study of Survey Data from 17 Countries. Social Science and Medicine 36:9, 1207-1227.

Black, R. E., G. Lopez de Romana, K. H. Brown, N. Bravo, O. G . Bazalar, and H. C. Kanashiro (1989) Incidence and Etiology of Infantile Diarrhea and Major Routes of Transmission in Huascar, Redu. American Journal of Epidemiology 129, 785-799.

Boerma, J. T., and George T. Bicego (1992) Preceding Birth Interval and Child Survival: Searching for Pathways of Influence. Studies in Family Planning 23:4, 243-256.

Caldwell, J. (1979) Education as a Factor in Mortality Decline: An Examination of Nigerian Data. Population Studies 33 :2, 395-413.

Caldwell. J, P. H. Reddy, and P. Caldwell (1983) The Social Components of Mortality: An Investigation in South India Employing Alternative Methodologies. Population Studies 37:2, 185-205.

Caldwell, John C. (1989) Mass Education as a Determinant of Mortality Decline. pp.101-111 In J. C. Caldwell and G. Santow (ed.) Selected Readings in the Cultural, Social and Behavioural Determinants of Health. Canberra: Health Transition Centre, The Australian National University.

Caldwell, John C. (1990) Cultural and Social Factors Influencing Mortality Levels in Developing Countries. Annals of the American Academy of Political and Social Science 510, 44-59.

Carlaw, R. W., and K. Vaidya (1983) Birth Intervals and the Survival od Children to Age Five: Some Data from Napal. Journal of Tropical Pediatrics 29:1, 31-34.

Cleland, J., and J. K.Van Ginneken (1988) Maternal Education and Child Survival in Developing Counties: The Search for Pathways of Influence. Social Science and Medicine 27, 1357-1368. 
Cleland, J, and Z. A. Sathar (1984) The Effect of Birth Spacing on Childhood Mortality in Pakistan. Population Studies 38: 3, 401-418.

Curtis, S. L., I Diammond, and J. W. McDonald (1993) Birth Interval and Family Effects on Post-neonatal Mortality in Brazil. Demography 30:1, 33-43.

Das Gupta, M. (1990) Death Clustering, Mother's Education and the Determinants of Child Mortality in Rural Punjab, India. Population Studies 449:3, 489-505.

Elegbe, I. A., O. O. Ojoteitimi, I. Elegbe, and M. O. Akinola (1982) Pathogenic Bacteria Isolated from Infant Feeding Teats. Contamination of Teats Used by Illiterate and Educated Nursing Mothers in Ile-Ife, Nigeria. American Journal of Diseases Child 136, 672-674.

Esrey, S. A. , and J. P. Habicht (1986) Epidemiologic Evidence for Health Benefits from Improved Water and Sanitation in Developing Countries. Epidemiological Review 8, 117-128.

Forste, Renata (1994) The Effects of Breast-feeding and Birth Spacing on Infant and Child Mortality in Bolivia. Population Studies 48, 497-511.

Gubhaju, B. (1985) The Effect of Previous Child Death on Infant and Child Mortality in Rural Nepal. Journal of Biosocial Science 16, 231-236.

Hobcraft, J. N., J. W. Mcdonald, and S. O. Rutstein (1985) Demographic Determinants of Infant and early Child Mortality: A Comparative Analysis. Population Studies 39, 363-385.

Hobcraft, J., J. W. Mcdonald, and S. O. Rutstein, (1984) Socio-economic Factors in Infant and Child Mortality: A Cross-national Comparison. Population Studies 38:2, 193-223.

Kimm, Sue, and G. Gonoso (1979) Control of Protein-calorie Malnutrition. In J. Mayer and T. Johanna Dwyer (eds.) Food and Nutrition Policy in a Changing World. New York: Oxford University Press.

Majumder, K. (1989) Factors Affecting Child Survival in Bangladesh. Australian Nationall University, Canberra. Unpublished Ph.D. dissertation.

Mosley, W., and Lincoln C. Chen (1984) Nalytical Framework for the Study of Child Survival in Developing Countries. Population Development Review, A Supplement to 10, 25-45.

Muhuri, P. (1995) Health Programmes, Maternal Education, and Differential Child Mortality in Matab, Bangladesh. Population and Development Review 21: 4, 813-834.

Pakistan, Government of (2000) Economic Survey of Pakistan 1999-2000. Islamabad: Planning Commission, Ministry of Planning and Development.

Phillips, L., S. K. Lwanga, W. Lore, and D. Wassawa (1969) Methods and Hygiene of Infant Feeding in an Urban area of Uganda. Journal of Tropical Pediatrics $15,167-171$. 
Rees, Jane M., Lederman, Sally A. Kiely, and L. John (1999) Neonatal Mortality among High Birth Weight Infants Born to African American Adolescent Mothers. Pediatric Research 45: 4.

Rutstein, Shea Oscar (1984) Infant and Child Mortality: Levels Trends and Demographic Differentials, Revised Edition. WFS Comparative Studies No. 43.

Ruzicka, L. T. (1989) Problems and Issues in the Study of Mortality Differentials. In Ruzicka, G. Wunsch and P.Kane (ed.) Differentials Mortality: Methodological Issues and Biosocial Factors. Oxford: Clarendon Press.

Sathar, Z. A., (1985) Infant and Child Mortality in Pakistan, Some Trends and Differentials. Journal of Biosocial Science 17:3, 351-359.

Sathar, Z. A. (1987a) Seeking Explanations for High Levels of Infant Mortality in Pakistan. The Pakistan Development Review 26:1.

Sathar, Z. A. (1988) Birth Spacing in Pakistan, Journal of Biosocial Science 20: 2, 175-94.

Sandiford P., Cassel J., Montenegro M., and G. Sanchez (1995) The Impact of Women's Literacy on Child Health and its Interaction with Access to Health Services. Population Studies 49: 1, 5-17.

Sullivan, J. M., George Bicego, and Shea Oscar Rutstein (1990) Assessment of the Quality of Data Used for the Direct Estimation of Infant and Child Mortality in the Demographic and Health Surveys an Assessment of DHS-I Data Quality. Maryland: Columbia. (DHS Methodological Reports No. 1.) 